\title{
Maximizing Photovoltaic Array Energy Usage Within a House Using Model Predictive Control
}

\author{
Aziz Ahmad \\ Department of Electrical \& \\ Electronics Engineering \\ Unitec Institute of Technology \\ Auckland, 1025, New Zealand \\ E-mail: aahmad@unitec.ac.nz
}

\author{
Timothy N. Anderson and Tek T. Lie \\ School of Engineering, Computer \& \\ Mathematical Sciences \\ Auckland University of Technology \\ Auckland, 1010, New Zealand \\ Email: timothy.anderson@aut.ac.nz
}

\author{
Akshya K. Swain \\ Department of Electrical \& Computer \\ Engineering \\ The University of Auckland \\ Auckland, 1023, New Zealand \\ Email: a.swain@auckland.ac.nz
}

\begin{abstract}
In this study, the problems of modeling, energy dispatching and Photovoltaic (PV) array energy priorities for a grid connected residential house with $P V$ array and battery storage using model predictive control (MPC) have been investigated. Artificial neural network (ANN) based global solar radiation forecast was used to plan in advance for periods of low sunshine. MPC was able to reduce electricity consumption in the house when solar radiation forecast was unfavorable. Quadratic programming optimization was used to maximize usage of the PV system. Excess energy from the PV array was used to further raise hot water cylinder (HWC) temperature, rather than exporting it to the utility grid. Performance of the overall model predictive control system was verified using simulation results.
\end{abstract}

Keywords-Building energy management, model predictive control, energy efficiency, photovoltaic energy systems, solar radiation forecast

\section{INTRODUCTION}

Climate changes, diminishing world supplies of the nonrenewable fuels as well as economic aspects are the driving factors of current effort to save the energy. Sustainable energy sources, such as wind, solar and their hybrid systems are becoming attractive options of providing energy globally for reasons such as low cost and easy accessibility [1]. Rapid development of global economy has remarkably increased energy requirements, especially in emergent countries. The realization that fossil fuel resources required for the generation of energy are depleting and that climate change is related to carbon emissions to the atmosphere has also increased interest in energy saving and environmental protection. The first strategy to reduce dependence on fossil resources is based on reducing power consumption by applying energy savings programs focused on energy demand reduction and energy efficiency in industrial and domestic applications [2].

To achieve the desired goal, renewable energy sources can be utilized, not only for large-scale energy production, but also for small-scale residential sector [3]. The employment of established passive technologies such as improved building insulation or more energy efficient appliances for heating and cooling are efficient methods towards the energy efficient operation of buildings. Another approach is to improve buildings automation by using advanced control concepts [4].
Current implementation of control processes in buildings still employ rule-based approaches combined with proportionalintegral-derivative (PID) controllers which operates in feedback arrangement and are prone to calibration errors and cannot handle unpredicted time delays. Also these PID controllers cannot handle nonlinearities in the control process and operate only in the predetermined time horizons.

The building dynamics are slow and the building is subject to intermittent disturbances, i.e. the weather as well as the energy consumption of building's appliances as well as the number of occupants, who generate heat, $\mathrm{CO}_{2}$, and set demands for temperature, illuminance and air quality. This gives rise to a constrained control problem and the goal is to use weather forecast in order to be able to make appropriate use of the thermal storage capacity of a building, electrical appliances and energy dispatch strategies. Model Predictive Control is an ideal framework to tackle this problem [5].

\section{LITERATURE REVIEW}

MPC is a method for constrained optimal control, which originated in the late seventies and early eighties in the process industries (oil refineries, chemical plants, etc.) [6], [7], [8], [9]. MPC is a class of control methods with the model of the process explicitly expressed in order to obtain a control signal by minimizing an objective function subject to some constraints. In case of building control, one would aim at optimizing the energy delivered (or cost of the energy) subject to comfort and the power system constraints.

Predictive control strategies for hybrid renewable energy systems are shown to be more efficient when compared to conventional, non-predictive strategies for energy efficient building automation [10], [11], [12]. In [13], demand side management of PV-battery hybrid systems using MPC approach has been discussed, in which the battery was charged from the grid during off-peak time and discharged during peak time to minimize grid imports during peak hours when electricity prices are usually higher. The proposed MPC achieved improved control performance in terms of accuracy and robustness. Furthermore, it was shown that more cost savings can be achieved by using the closed-loop control. The grid-connected PV-battery-grid system can help customers, to reduce energy 
imports from the local grid, and also can help utility grid to regulate the grid in terms of security and efficiency issues, such as peak shaving, direct load control (DLC), and capacity market programs [14].

Some attempts have been made to test MPC for small scale distributed power generation systems including the work of [15], which proposed a supervisory control system using MPC. The controller focused on the optimal management and operation of the small-scale hybrid wind-solar energy systems. Two local controllers were used to drive the two subsystems to the power references and MPC capability to reduce the peak values of inrush or surge currents was discussed.

To achieve maximum efficiency out of photovoltaic systems using small-scale batteries and flexible thermal loads, [16] proposed four rule-based control algorithms and calculated the building energy flows and PV self-consumption ratios on an annual basis. Battery capacities that maximize the savings over the investment lifetime for different combinations of battery capital costs and PV feed-in tariffs have been investigated.

In [17], a model predictive control strategy was utilized to maximize PV self-consumption, exploiting the flexible demand of an electric water heater. Solar irradiance forecast and real PV production data was used to test the system. Simulations showed the ability of MPC to move the consumption of the heater to a time when there was energy production from the PV system.

To find a generalized DSM strategy based on load shifting, a heuristic-based evolutionary algorithm was developed in [18]. In [19], neural network based approach was used to schedule and coordinate distributed generations for active demand side management.

In [20], an optimal load management strategy for residential households was studied, which utilized the communication infrastructure of the future smart grid. Prediction of electricity prices, energy demand, PV production and consumer's powerpurchase of energy were utilized to determine the optimal relationship between hourly electricity prices and the use of different household appliances for a typical smart house. Results showed that the proposed model allowed users to reduce their electricity bill between $8 \%$ and $22 \%$ for the typical summer day analyzed and adapt the electricity bill to their actual economic situation. An advance control strategy for a smart home was developed in [18], to enable consumer economic saving and automate the demand side management for domestic users. Another event driven controller was developed in [21], for the optimal scheduling of household appliances using binary linear programming.

Another attempt to use linear predictive control approach to reduce the use of auxiliary energy demand and increase solar yields by using weather forecast data, was made by [22], which demonstrated the feasibility of the proposed approach for a solar thermal water tank with auxiliary heating elements for a residential house.

MPC has been widely used in the closed-loop control for adaptively changing control variables according to external disturbances and is applied in this work because of its ability to explicitly handle constraints and to adjust the energy flows when disturbances occur.

\section{METHODOLOGY}

The proposed controller will be able to forecast the availability and magnitude of the solar resource to plan in advance for periods when the solar radiation magnitude is small or unavailable and should be adaptable to a range of timescales and locations.

\section{A. Global solar radiation forecast}

In [23], several forecasting techniques were analyzed: NARX, MLP, ARMA and persistence. Hourly time series data for nine historic weather variables recorded over a three-year period were used to train and test the forecasting methods for New Zealand's largest city, Auckland. Results from forecasts based on the NARX were compared with an ANN based Multilayer Perceptron (MLP) method, a statistical approach using auto regressive moving average (ARMA) and a reference persistence approach. Predicted values of hourly global solar irradiation were compared with the measured values, and it was found that the root mean squared error (RMSE) was 0.243 $\mathrm{MJ} / \mathrm{m}^{2}$ for the NARX method as compared to $0.484 \mathrm{MJ} / \mathrm{m}^{2}$, $0.315 \mathrm{MJ} / \mathrm{m}^{2}$ and $0.514 \mathrm{MJ} / \mathrm{m}^{2}$ for the MLP, ARMA and persistence approaches respectively. Subsequently the NARX approach was used to forecast global solar irradiation for other major cities across New Zealand. The results demonstrated the ability of the NARX approach to forecast irradiation values at a later time and across a number of different locations.

Figure 1 shows a randomly selected one week forecast of global solar irradiation in Auckland using hourly time series data for nine historic weather variables recorded over a three-year period using the proposed NARX approach. It can be seen that over this single week, that the NARX forecasts the solar irradiation with a high degree of accuracy.

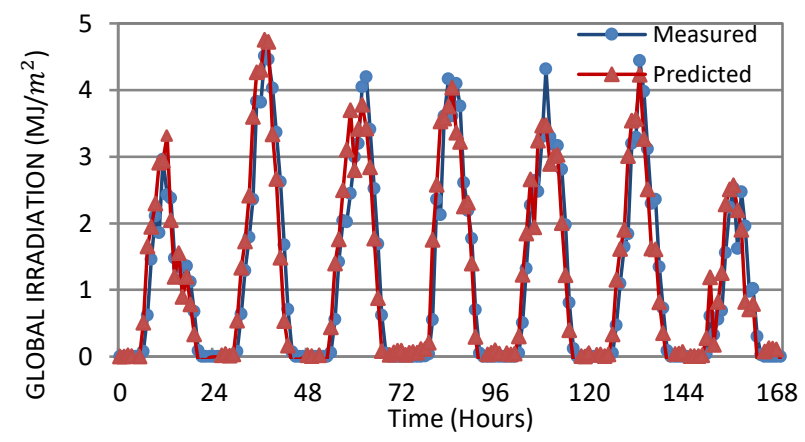

Figure 1. One-week measured and predicted solar radiation values using NARX approach

Further, the NARX architecture was used to successfully forecast global solar irradiation in ten major cities across New Zealand. These results have demonstrated the generalization capability of this approach and its ability to produce accurate forecasts for global irradiation that can be translated to a number of diverse locations. On this basis it is conceivable that such a NARX ANN forecasting approach could be embedded into 
model predictive controllers to better manage the energy generated by solar energy systems.

\section{B. Structure of the photovoltaic based power generation system}

Figure 3 shows the overall structure of the proposed grid connected house with PV array and battery storage. Electrical loads of the house are divided into critical (hot water cylinder, lighting, power sockets, fridge and cooking range) and noncritical (dish washer, washing machine and dryer) loads.

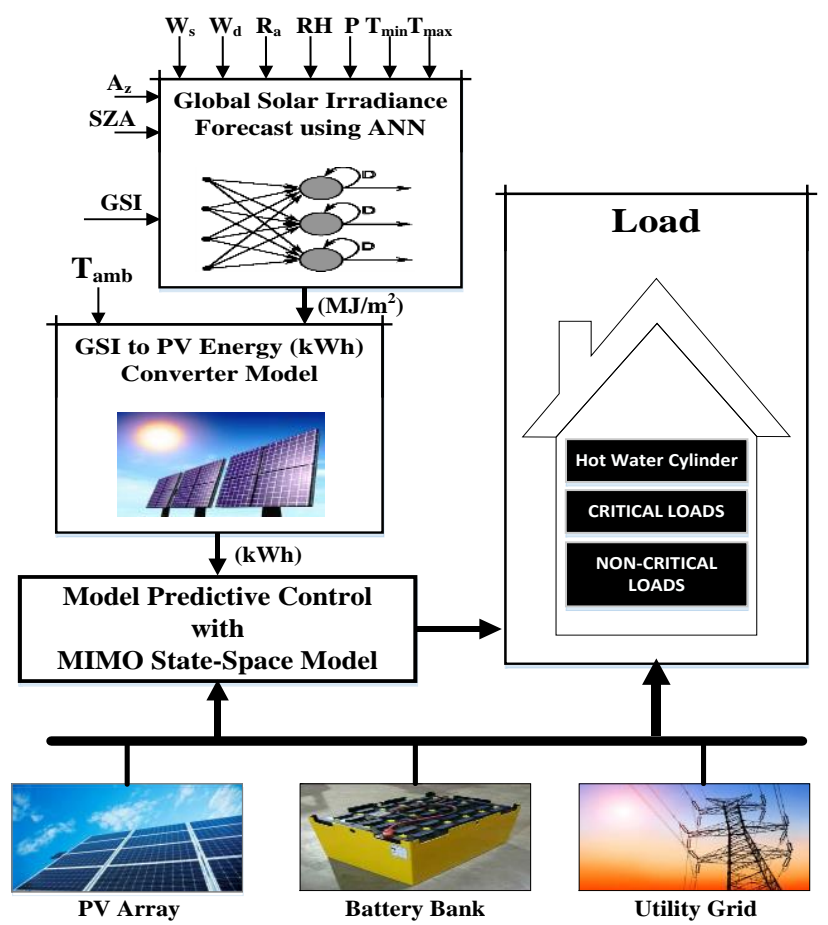

Figure 1. Structure of the photovoltaic-battery-grid system for a residential house

One-hour ahead global solar radiation forecast is calculated as discussed in the previous section. This forecast is made available to the MPC to activate or deactivate non-critical loads of the house to adjust energy demand according to the availability or unavailability of the PV energy. MPC uses the PV array production as reference signal and attempting to minimize the difference between electricity consumption in the house and PV energy production. The objective of the controller was to maximize the usage of PV energy within the house rather than exporting all available excess energy to the grid.

Figure 4 shows energy dispatching for the proposed grid connected house with PV array installation and battery storage. Energy from the PV array, battery bank and grid are shown to satisfy energy demand of the house. The arrows in Figure 4 show direction of energy flow in the system. The output energy of the PV array is used to satisfy energy demand of the house and charge the battery bank. At any given hour, $80 \%$ of PV energy $\left(E_{P V 1}\right)$ flows to satisfy energy demand of the house and $20 \%$ of PV energy $\left(E_{P V b}\right)$ to charge the battery bank. When the battery bank is fully charged and excess PV energy is available, the hot water temperature in the hot water cylinder is increased to the maximum threshold using $E_{P V 2}$. Further, if PV array is still producing more energy at any given hour, it is exported to the grid as a last priority represented by $E_{P V 3}$. If energy demand of the house is larger than the PV array production, the deficient energy can be covered by the battery bank using $E_{b}$ or the grid energy $E_{g}$ in the case where the battery bank is fully depleted.

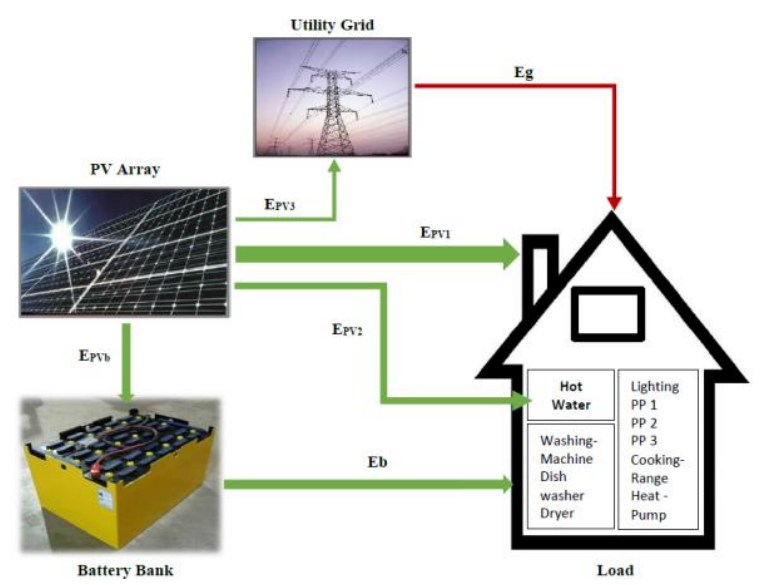

Figure 4. Configuration of the photovoltaic-battery-grid energy system

Several priorities are specified in the MPC design for the PV array energy. $E_{P V 1}$ is the PV energy to satisfy energy demand of the house with first priority, $E_{P V 2}$ is the PV energy to increase hot water cylinder temperature to its maximum threshold with second priority and $E_{P V 3}$ is the PV energy flowing to the grid with third priority. $E_{P V b}$ is the PV energy to charge the battery bank, $E_{b}$ is battery bank energy to satisfy energy demand of the house when energy from the PV array is not sufficient and $E_{g}$ is the energy flowing from the grid whenever, energy from both the PV array and the battery bank is not sufficient to satisfy energy demand of the house.

\section{Model Predictive Control Design}

MPC systems are designed based on a mathematical model of the plant. In this work multi input multi output (MIMO) statespace model has been used for the MPC design. By using a statespace model, the current information required for predicting ahead is represented by the state variable at the current time.

\section{A. MIMO state-space model of the proposed system}

The proposed plant has five inputs, 3 outputs and four states. The number of outputs are less than the number of inputs, therefore, each measured output can be controlled independently with zero steady-state errors. Taking the disturbance into consideration, the predictive control problem can be formulated as

$$
\begin{gathered}
x_{m}(k+1)=A_{m} x_{m}(k)+B_{m} u(k)+D_{m} \omega(k) \\
y(k)=C_{m} x_{m}(k)
\end{gathered}
$$


Where $\omega(k)$ is the measured input disturbance which essentially represent the ANN based one-hour ahead solar radiation forecast. $u$ is the manipulated variable or input variable; $y$ is the process output; and $x_{m}$ is the state variable vector. $A_{m}$ is the state matrix, $B_{m}$ is the input-to-state matrix, $C_{m}$ is the state-to-output matrix and $D_{m}$ is the direct feed-through matrix of the state-space model.

\section{B. Solution of the predictive control}

Output vectors $Y$ and input vectors $\Delta U$ are defined in Equations (3) and (4) respectively.

$$
\begin{gathered}
\Delta U=\left[\begin{array}{c}
\Delta u\left(k_{i}\right)^{T} \Delta u\left(k_{i}+1\right)^{T} \Delta u\left(k_{i}+2\right)^{T} \ldots \ldots \\
\Delta u\left(k_{i}+N_{c}-1\right)^{T}
\end{array}\right]^{T} \\
Y=\left[\begin{array}{c}
y\left(k_{i}+1 \mid k_{i}\right)^{T} y\left(k_{i}+2 \mid k_{i}\right)^{T} \\
y\left(k_{i}+3 \mid k_{i}\right)^{T} \ldots \ldots y\left(k_{i}+N_{p} \mid k_{i}\right)^{T}
\end{array}\right]^{T}
\end{gathered}
$$

Now from the state-space model (A, B, C, D), the predicted state variables are computed sequentially using the set of future control parameters as:

$$
\begin{aligned}
& x\left(k_{i}+1 \mid k_{i}\right)=A x\left(k_{i}\right)+B \Delta u\left(k_{i}\right)+B_{d} \epsilon\left(k_{i}\right) \\
& x\left(k_{i}+2 \mid k_{i}\right)=A x\left(k_{i}+1 \mid k_{i}\right)+B \Delta u\left(k_{i}+1\right) \\
&+B_{d} \epsilon\left(k_{i}+1 \mid k_{i}\right)
\end{aligned}
$$

With the assumption that $\epsilon(k)$ is a zero-mean white noise sequence, the predicted value of $\epsilon\left(k_{i}+i \mid k_{i}\right)$ at future samples $i$ assumed to be zero. For notational simplicity, above equations can be collected together in a compact matrix from as

$$
Y=F x\left(k_{i}\right)+\Phi \Delta U
$$

where

$$
\begin{gathered}
F=\left[\begin{array}{c}
C A \\
C A^{2} \\
C A^{3} \\
\vdots \\
C A^{N_{p}}
\end{array}\right] ; \\
\Phi=\left[\begin{array}{ccccc}
C B & 0 & 0 & \cdots & 0 \\
C A B & C B & 0 & \cdots & 0 \\
C A^{2} B & C A B & C B & \cdots & 0 \\
\vdots & \vdots & \vdots & \cdots & \vdots \\
C A^{N_{p}-1} B & C A^{N_{p}-2} B & C A^{N_{p}-3} B & \cdots & C A^{N_{p}-N_{C} B}
\end{array}\right]
\end{gathered}
$$

where dimensions of $Y$ is $N_{p} p \times 1, x$ is $n \times 1, F$ is $N_{p} p \times n, \Phi$ is $N_{n} p \times N_{c} m$ and $\Delta U$ is $N_{c} m \times 1$. The control calculations are based on minimizing the predicted deviations between the predicted output and the reference trajectory. The predicted error vector is defined as

$$
\widehat{E}\left(k_{i}+1\right)=Y_{r}\left(k_{i}+1\right)-Y\left(k_{i}+1\right)
$$

This is $N_{p} p \times 1$ vector. The objective of the control calculations is to determine the control moves $\Delta U\left(k_{i}\right)$ for the next $N_{c}$ time intervals. The $N_{c} m$-dimentional vector $\Delta U\left(k_{i}\right)$ is calculated such that an objective function is minimized. Also the predicted error over the prediction horizon $N_{p}$ and the size of the control move over the control horizon $N_{c}$ is minimized.

\section{Optimization}

The objective of the predictive control system for a given reference signal $r\left(k_{i}\right)$ at sample time $k_{i}$, within a prediction horizon $N_{p}$ to bring the predicted output as close as possible to the reference signal. To find the optimum control parameter vector $\Delta U$, the objective is translated into a design such that the error function between the reference signal and the predicted output is minimized as shown in Equation (a16). The cost function $J$ which reflects the control objective is defined as

$$
J=\left(R_{s}-Y\right)^{T}\left(R_{s}-Y\right)+\Delta U^{T} \bar{R} \Delta U
$$

where the first term is linked to the objective of minimizing the errors between the predicted output and the reference signal while the second term reflects the consideration given to the size of $\Delta U$ when the objective function $J$ is made to be as small as possible.

\section{Constraints for the MIMO state-space model}

Constraint variables are parametrized using the same parameter vector $\Delta U$ as the one used in the predictive control design. Therefore, the constraints are expressed in a set of linear equations based on the parameter vector $\Delta U$. Constraints are categorized as follow. As the cost function is quadratic and constraints are linear inequalities, the solution for the optimal predictive control is similar to finding an optimal solution for a standard quadratic programming problem. For compactness of expression, Equation (a33) can be expressed as

$$
M \Delta U \leq \gamma
$$

Where $M$ is a matrix representing constraints. The number of rows and columns in the matrix $M$ is equal to the number of constraints and the dimension of $\Delta U$ respectively.

$$
M=\left[\begin{array}{ccccc}
-1 & 0 & 0 & 0 & 0 \\
0 & -1 & 0 & 0 & 0 \\
0 & 0 & -1 & 0 & 0 \\
0 & 0 & 0 & -1 & 0 \\
0 & 0 & 0 & 0 & -1 \\
1 & 1 & 0 & 0 & 1 \\
1 & 1 & 1 & 1 & 0 \\
1 & 0 & 0 & 0 & 0 \\
0 & 1 & 0 & 0 & 0 \\
0 & 0 & 1 & 0 & 0 \\
0 & 0 & 0 & 1 & 0 \\
0 & 0 & 0 & 0 & 1 \\
-1 & -1 & 0 & 0 & -1
\end{array}\right], \quad \gamma=\left[\begin{array}{c}
0 \\
0 \\
0 \\
0 \\
0 \\
E_{d}(k) \\
E_{P V}(k) \\
E_{P V 1}^{\max } \\
E_{P V 2}^{\max } \\
E_{P V 3}^{\max } \\
E_{P V b}^{\max } \\
E_{g}^{\max } \\
E_{g}^{\max }-E_{d}(k)
\end{array}\right]
$$


Constraints for the state-of-charge of the battery should be in the form similar to the predictive control vector $U(k)$. Predicted values of $x_{m}$ for the SOC can be calculated by

$$
S_{o c}(k+i \mid k)=S_{o c}(k)+b_{m} \sum_{j=k}^{j \leq k+i-1} u(j)
$$

where $S_{o c}(k+i \mid k)$ is the predicted value of $S_{o c}$ from sampling time $k$.

To avoid charging and discharging the battery simultaneously, $E_{P V b}=0$ can be set for charging and $E_{b}=0$ can be set for discharging. The procedure mentioned above are for charging. For discharging row 4 is replaced by

$$
M=\left[\begin{array}{lllll}
0 & 0 & 0 & 1 & 0
\end{array}\right], \quad \gamma=[0]
$$

MPC is developed for the closed-loop control, in which the objective function for the plant model is to maximize the utilization of PV energy within the house and differ usage of non-critical loads in the house to periods when excess PV energy is available by utilizing solar radiation forecast. The objective function over the prediction horizon $T_{N}=\left[k, k+N_{p}\right],(k \geq 0$ is an integer) is given by

$$
J(k)=\sum_{k}^{k+N_{p}}\left\{\left[\begin{array}{c}
E_{P V}(k)-w_{2} E_{P V 1}(k)-w_{2} E_{P V 2}(k) \\
-w_{2} E_{P V 3}(k)-w_{2} E_{P V b}(k)
\end{array}\right]^{2}\right\}
$$

where $N_{p}$ represent hours over the prediction horizon for the MPC design.

\section{Simulation Results And Discussion}

In this section, simulation results of the proposed system are presented. The simulation was undertaken using a 24 hours measurements of PV array production $\left(E_{P V}(k)\right)$ and energy demand $\left(E_{d}(k)\right)$ taken from a real house, as shown in Figure 5. Total energy demand in Figure 5 represent critical loads (hot water cylinder, lighting, power sockets, fridge and cooking range) and non-critical loads (dish washer, washing machine and dryer) combined.

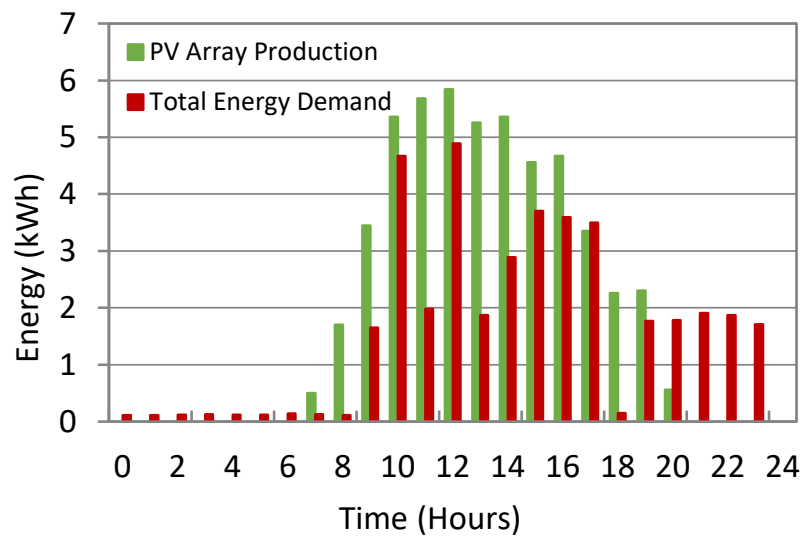

Figure 5. PV array production and total energy demand of the house
Figure 6 shows switching behavior of the MPC in the presence of positive or negative disturbance. If forecasted PV energy differs significantly than the current PV energy production, non-critical loads remain off to reduce total load of the house. To avoid switching due to small weather variations, a weighting factor is added to reduce switching sensitivity. For example, if predicted PV energy at any hour is 1.5 times more than the current PV energy, non-critical loads are turned off.

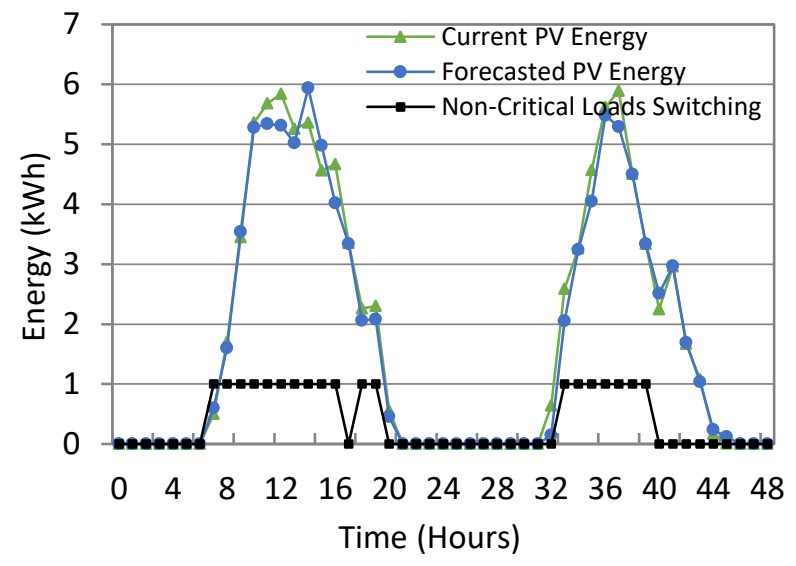

Figure 6. MPC performance in the presence of disturbance (PV energy prediction $)(\mathrm{On}=1, \mathrm{Off}=0)$

Further, the performance of the proposed MPC was tested by analyzing how closely the output of the controller followed the reference signal. From the objective function, it can be deduced that minimizing the difference between the controller output and the reference signal is equivalent to maximizing PV energy usage within the house. In Figure 7 it can be seen that the MPC is attempting to minimize the difference between the controller output signal and the reference signal. which is equivalent to maximizing the usage of the PV array energy within the house and consequently helping reduce grid imports.

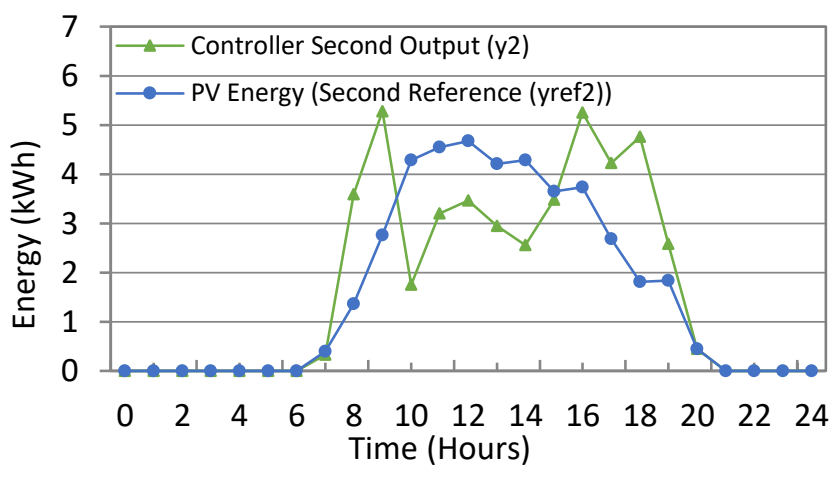

Figure 7. Controller output signal 2 vs reference signal 2

To analyze the cost saving potential of the proposed MPC approach, one houses with the same PV array installation, battery storage and grid connection is using the proposed controller with solar radiation forecast and the other house is 
using the same setup without the controller. Table 1 show grid import/export for both the houses. It can be seen that the proposed controller imports less energy from the grid and consequently saving cost on electricity for the user.

TABLE I. ONE WEEK SAVING COMPARISON FOR THE HOUSE WITH MPC CONTROL AND A HOUSE WITHOUT CONTROL

\begin{tabular}{|c|c|c|c|}
\hline $\begin{array}{c}\text { House with } \\
\text { MPC. Grid } \\
\text { Exports (kWh) }\end{array}$ & $\begin{array}{c}\text { House with } \\
\text { MPC. Grid } \\
\text { Imports (kWh) }\end{array}$ & $\begin{array}{c}\text { House with No } \\
\text { Control. Grid } \\
\text { Exports (kWh) }\end{array}$ & $\begin{array}{c}\text { House with No } \\
\text { Control. Grid } \\
\text { Imports (kWh) }\end{array}$ \\
\hline $\mathbf{8 9 . 2 8}$ & $\mathbf{1 6 5 . 5 3}$ & $\mathbf{7 3}$ & $\mathbf{1 9 8}$ \\
\hline
\end{tabular}

\section{CONCLUSION}

In this study, adaptive MPC strategy was developed for energy dispatching of a grid connected residential house with PV array installation and battery storage. Artificial neural network based solar radiation forecast was used as a measured disturbance, to plan in advance for periods of low sunshine, which represents lower PV energy production. The model predictive controller was found to be capable of operating noncritical loads when excess PV energy was available and also was able to dispatch energy to and from the battery storage when needed. Excess energy from the PV array was utilized to further raise hot water temperature in the tank, rather than exporting it to the utility grid. Two houses with the same PV array installation and battery storage, but one with the proposed controller and another without a controller were used to compare the cost saving potential of the proposed approach. It was observed that, the MPC approach imports $32.47 \mathrm{kWh}$ less energy from the grid within a one-week period as compared to the house without a controller. Also, MPC approach managed to export $16.28 \mathrm{kWh}$ more energy to the grid as compared to a house without a controller using the same PV production and energy demand data.

\section{REFERENCES}

[1] T. Hove, and H. Tazvinga, "A techno-economic model for optimising component sizing and energy dispatch strategy for PV-diesel-battery hybrid power systems", Journal of Energy in Southern Africa, vol. 23, pp. 18-28, 2012.

[2] M. Martiskainen and J. Coburn, "The role of information and communication technologies in household energy consumption-prospects for the UK", Energy Efficiency, vol. 4, pp. 209-221, 2011.

[3] W. Zhou, C. Lou, Z. Li, 1. Lu and h. Yang, "Current status of research on optimum sizing of stand-alone hybrid solar-wind power generation systems", Applied Energy, vol. 87, pp. 380-389, 2010.

[4] J. Laustsen, "Energy efficiency requirements in building codes, energy efficiency policies for new buildings", International Energy Agency, IEA Information paper, OECD/IEA, 2008, pp. 1-85.

[5] F. Oldewurtel, A. Parisio, N. C. Jonesc, D. Gyalistrasa, M. Gwerderd, V. Stauche, B. Lehmannf and M. Moraria, "Use of model predictive control and weather forecasts for energy efficient building climate control", Energy and Buildings, 2012, vol. 45, pp. 15-27
[6] J. Richalet, J. A. Rault, L. Testud \& J. Papon, 1976, “Algoritmic control of industrial process", Proceedings: Symposium on identification and system parameter estimation. IFAC. Tbilisi, 1976

[7] M. J. Maciejowski, 2002, "Predictive control with constraints", Pearson Education Limited

[8] J. Richalet, A. Rault, J. Testud \& J. Papon, 1978, "Model predictive heuristic control: Applications to industrial processes", Automatica, vol. 14, pp. 413-428

[9] W. Kwon, A. Bruckstein \& T. Kailath, 1983, "Stabilizing state-feedback design via the moving horizon method", International Journal of Control, vol. 37, pp. 631-643

[10] D. C. Bourgeois \& I. Macdonald, 2006, “Adding advanced behavioural models in whole building energy simulation: a study on the total energy impact of manual and automated lighting control", Energy and Buildings, vol. 38, pp. 814-823

[11] S. Cho \& M. Zaheer-uddin, 2003, "Predictive control of intermittently operated radiant floor heating systems", Energy and Conversion Management, vol. 44, pp. 1333-1342

[12] G. Henze, D. Kalz, D, Liu, S. \& Felsmann, C., 2005, "Experimental analysis of model-based predictive optimal control for active and passive building thermal storage inventory", International Journal of HVAC \& Research, vol. 11, pp. 189-214

[13] Z. Wu, H. Tazvinga \& X. Xia, 2015, "Demand side management of photovoltaic-battery hybrid system", Applied Energy, vol. 148, pp. 294 304

[14] H. Aalami, P. M. Moghaddam \& G. Yousefi, 2010, "Demand response modelling considering interruptible/curtailable loads and capacity market programs", Applied Energy, vol. 87, pp. 243-250

[15] J. Lagorse, M. Simoes, A. Miraoui, "A multiagent fuzzy-logic-based energy management of hybrid systems", IEEE Transactions on Industrial Applications, 2009, vol. 45, pp. 2123-2129

[16] J. Torreglosa, P. Garca, L. Fernndez, F. Jurado, "Hierarchical energy management system for stand-alone hybrid system based on generation costs and cascade control", Energy Conversion and Management, 2014, vol. 77, pp. 514-526

[17] H. Morais, P. Kádár, P. Faria, Z. A. Vale, H. Khodr, “Optimal scheduling of a renewable micro-grid in an isolated load area using mixed-integer linear programming", Renewable Energy, 2010, vol. 35(1), pp. 151-156

[18] J. M. Duran, J. Prieto, F. Barrero and S. Toral, "Predictive current control of dual three-phase drives using restrained search techniques", IEEE Transaction on Industrial Electronics, 2011, vol. 58, pp. 3253-3263

[19] F. Barrero, J. Prieto, E. Levi, R. Gregor, S. Toral, J. M. Duran and M. Jones, "An enhanced predictive current control method for asymmetrical six-phase motor drives", IEEE Transaction on Industrial Electronics, 2011, vol. 58, pp. 3242-3252

[20] S. Thomsen, N. Hoffmann and W. F. Fuchs, "PI control, PI-based state space control and model-based predictive control for drive systems with elastically coupled loads-A comparative study", IEEE Transaction on Industrial Electronics, 2011, vol. 58, pp. 3647-3657

[21] A. Mohsenian-Rad and A. Leon-Garcia, "Optimal residential load control with price prediction in real-time electricity pricing environments", IEEE Transactions on Smart Grid, 2010, vol. 1, pp. 120-133

[22] M. F. Pichler, W. Lerch, A. Heinz, G. Goertler, H. Schranzhofer \& R. Rieberer, 2014, "A novel linear predictive control approach for auxiliary energy supply to a solar thermal combistorage", Solar Energy, vol. 101, pp. 203-219

[23] A. Ahmad, T. N. Anderson, and T. T. Lie, "Global solar irradiation forecast for New Zealand”, Solar Energy, 2015, Vol. 122, Pp. 1398-1408 